\title{
A Review on Nanomedicine as a Modern Form of Drug Delivery
}

\author{
Ahmad N*, Khan Z and Hasan N \\ Faculty of pharmacy, Integral University, India
}

Submission: March18, 2017; Published: April 27, 2017

*Corresponding author: Nesar Ahmad, Research Scholar, Faculty of pharmacy, Integral University, Tel: +919807273314, Fax: 0522-2890809; Email: nesar50@gmail.com

\begin{abstract}
Today nanomedicine is the method of treating, diagnosing, and preventing the disease and preserving and improving human health, using molecular tools and molecular knowledge of the human anatomy. Nanomedicines play exclusive and pivotal role in drug delivery, imaging and diagnosis. There are many simple nano devices can be manufactured for improving the medicals problems. Drug delivery systems can be used to in particular target to the tumors and improves the therapeutic index and pharmacokinetic profile of anticancer drugs. Many of the drugs have poor pharmacokinetics by this the nanomedicines will improve or to control the drug pharmacokinetics in human body. The goal of the nanomedicine is to detect changes and problems on the molecular and cellular levels and to treat them before they spread out the disease.
\end{abstract}

Keywords: Disease; Nanomedicine; Pivotal, Pharmacokinetics

\section{Introduction}

Nanomedicine is different from the conventional approaches in sense that conventional medicine's approach is based on a response to problems at a tissue level and by the time that symptoms become apparent to patient or clinician, the disease may be often at an advanced stage. Conventional technology may also rely more on the use of sophisticated and expensive medical tools, machines, robots, minimally invasive devices, and implants. However, in nanomedicine, the goal is to detect changes and problems on the molecular and cellular levels and to treat them before they spread out [1]. Cancer is the first leading cause of diseased associated death in China [2] and second leading cause of death in the United States [3] in recent years.

The recent treatment modalities for tumors which mainly include surgery, chemotherapy, radiotherapy, and immunotherapy, among which drug treatment is still most widely used therapeutic mode for various types of the tumors [4]. Cancer is not one, but many heterogeneous diseases characterized by the rapid and uncontrolled cellular expansion as a result the genetic and epigenetic alterations. It annually affects millions of people worldwide [5]. Present therapies for cancer include surgery, radiotherapy, chemotherapy and immunotherapy. Early diagnosis of the tumors facilitates to the treatment of patients with surgery and radiotherapy, however, in patients with tumors that cannot be resected or irradiated, the only available treatment options are chemotherapy and immunotherapy [6].

Drug delivery systems can be used to specifically target to the tumors and improves the therapeutic index and pharmacokinetic profile of anticancer drugs. Nanomedicines are submicrometer sized carrier materials which can intend to improve the bio distribution of the systemically administered chemotherapeutic drugs. Nano formulation aims that to improving the balance between the efficacy and toxicity of systemic chemotherapeutic agent interventions [7]. Among others, the chief examples of nanomedicine formulations are liposomes [8], micelles [9], nanoparticles [10] and polymer-drug conjugates [11].

\section{Nanotechnology applications in medicine}

Today drug delivery is one of the most common uses of nano particles (NPs) in the delivery and controlled release of therapeutic molecules. Nano materials contain engineered to fit in small molecule drugs as well as macro molecules such that nucleic acids, proteins, and peptides. One advantage of encapsulating therapeutic drugs in nano-particles includes the suspension of otherwise insoluble drugs [12]. Perhaps the greatest promise of nanomedicines for drug delivery 


\section{Global Journal of Nanomedicine}

applications is ability to control the drug pharmacokinetics [13]. Many potentially therapeutic molecules exhibit have poor pharmacokinetics, especially with respect to the kidneys. Various small molecule therapies are cleared from body by the renally, but their persistence in the kidneys is too brief to achieve therapeutic effects. Those Compounds that are cleared by hepato biliary mechanisms may have even less exposure to the kidneys.

The targeting of nano particles to specific organs, tissues, and cells in the body would be allow to the reduction of off-target side effects of drugs that produce toxicities when administered conventionally. The physical targeting of drugs may also enable to reduction in the total administered dose, as more pay load is available at the disease site instead of the healthy organs. Targeting is often achieved through the use of molecular recognition entities such that antibodies, peptides, nucleic acids, or small molecules, which may be attached to coat nano particles [13]. Nanomaterials incorporating therapeutic molecules can be engineered to the control rate of drug release. Nanomedicines can administer in depot format, allowing timed to release and single-dose administrations to achieve an optimal therapeutic index [14]. Also, the nano materials incorporating therapeutic molecules can be engineered to the control rate of drug release.

Nanomedicines can be administer in depot format, allowing timed to release and single dose administration to achieve an optimal therapeutic index can protect encapsulated molecules from degradation in body. This strategy can be combined with encapsulation, conjugation of therapeutic or imaging pay loads to design functional material, other therapeutic mechanism. Nano particles can also have other desirable therapeutic properties. Among these this is the potential for photo thermal therapy, often in oncology applications. Nano materials which composed of certain metals including gold and silver can produce an increase in local temperature because of the light-induced plasmon resonance - a physical property that can be only exploited at nano scale dimensions [15]. Such type plasmonic nano materials are often targeted by molecular recognition entities to the specific tissues before exposure to light, which producing a temperature elevation to kill the targeted cells. Another potential therapeutic use of nano materials is the selective sequestration and removal of the toxic substances, which often through the use of magnetic nano materials $[16,17]$.

\section{References}

1. Roa W, Zhang X, Guo L, Shaw A, Hu X, et al. (2009) Gold nanoparticles sensitize radiotherapy of prostate cancer cells by regulation of the cell cycle. Nanotechnology 20(37): 375101.

2. Chen W, Zheng R, Baade PD, Zhang S, Zeng H, et al. ( 2015) Cancer statistics in China. CA Cancer J Clin 66(2): 115-132.

3. Olaku 00, Taylor E (2017) Cancer in the Medically Underserved Population. Prim Care 44(1): 87-97.

4. Xiao B, Ma L, Merlin D (2017) Nanoparticle-mediated co-delivery of chemotherapeutic agent and siRNA for combination cancer therapy. Expert Opin Drug Deliv 14(1): 65-73.

5. Siegel RL, Miller KD, Jemal A (2016) Cancer statistics 2016. CA Cancer J Clin 66(1): 7-30.

6. Cho K, Wang X, Nie S, Chen ZG, Shin DM (2008) Therapeutic nanoparticles for drug delivery in cancer. Clin Cancer Res 14(5): 13101316.

7. Lammers T, Kiessling F, Hennink WE, Storm G (2002) Drug targeting to tumors. Principles, pitfalls and (pre-) clinical progress. J Control Release 161(2): 175-187.

8. Malam Y, Loizidou M, Seifalian AM (2009) Liposomes and nanoparticles. nanosized vehicles for drug delivery in cancer. Trends Pharmacol Sci 30(11): 592-599.

9. Park HS, Kim HA, Jung JW, Kim YK, Lee SK (2003) Metalloproteinase-9 is increased after toluene diisocyanate exposure in the induced sputum from patients with toluene diisocyanate induced asthma. Clin Exp Allergy 33(1): 113-118.

10. Hillaireau H, Couvreur P (2009) Nanocarriers' entry into the cell Relevance to drug delivery. Cell Mol Life Sci 66(17): 2873-2896.

11. R Duncan (2006) Polymer conjugates as anticancer nanomedicines. Nat Rev Cancer 6(9): 688-701.

12. Singh R, Lillard JW (2009) Nanoparticle-based targeted drug delivery. Exp Mol Pathol 86(3): 215-223.

13. Duncan R, Gaspar R (2011) Nanomedicine (s) under the microscope. Mol Pharm 8(6): 2101-2141.

14. Wang AZ, Langer R, Farokhzad OC (2012) Nanoparticle delivery of cancer drugs. Annu Rev Med 63:185-198.

15. Peer D, Karp JM, Hong S, Farokhzad OC, Margalit R, et al. (2007) Nanocarriers as an emerging platform for cancer therapy. Nat Nanotechnol 2(12): 751-760.

16. Sayed EIH, Huang X, Sayed EMA (2006) Selective laser photo-thermal therapy of epithelial carcinoma using anti-EGFR antibody conjugated gold nanoparticles. Cancer Lett 239(1): 129-135.

17. Hu CM, Fang RH, Copp J, Luk BT, Zhang L (2013) A biomimetic nanosponge that absorbs pore-forming toxins. Nat Nanotechnol 8(5): 336-340.

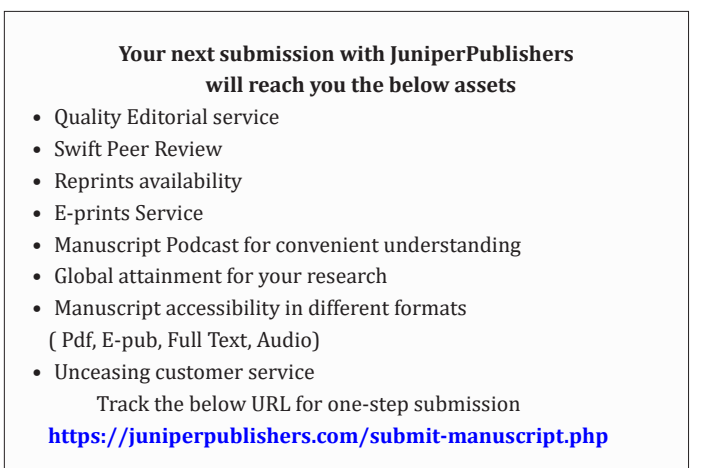

